\title{
Robust chaos revisited
}

\author{
Paul Glendinning ${ }^{\mathrm{a}}$ \\ School of Mathematics, University of Manchester, Oxford Road, Manchester M13 9PL, UK
}

Received 16 February 2017 / Received in final form 4 April 2017

Published online 21 June 2017

\begin{abstract}
Robust chaos is an important idea in the study of piecewise smooth maps. The different techniques used to prove the existence of robust chaos are reviewed and a new genericity condition for the classic example is established. The theoretical conditions for the existence of robust chaos are verified numerically providing additional evidence for robust chaos in some examples. This provides a new set of tools for the investigation of robust chaos.
\end{abstract}

\section{Introduction}

In a seminal paper, Banerjee, Yorke and Grebogi [4] introduced the idea of robust chaos in the context of piecewise linear maps of the plane. The idea has been useful in the analysis of many examples of piecewise smooth systems, but there is still some uncertainty about precisely what has been established. In their book on the bifurcations of piecewise smooth systems di Bernardo et al. note that '... additional (possibly generic) conditions must be true in order to guarantee that such a scenario definitely occurs, and the precise enumeration of the parameter region in which the chaotic attractor occurs remains an open problem.' ([5], pp. 157-158.) It seems remarkable that despite the influence of the original papers very little appears to have been done to determine what is known. The aim of this article is to consider the different approaches to robust chaos and to discuss the extent to which the mechanisms are fully understood and in particular (Lemma 2) another generic condition required for the arguments of Banerjee et al. [3,4] to hold is identified. This will involve revisiting not only the work of Banerjee et al. [3,4], but also important contributions from Misiurewicz [16] and Young [24]. These provide rigorous results that can be applied to examples, and in the final sections of this paper we adapt these to obtain numerical confirmation of the assumptions of Young's Theorem, and hence establish (up to the accuracy of the numerics) the existence of robust chaos by other means.

Contrary to common practice in mathematics papers I will discuss what I do not understand as well as what I do understand. This means that some questions arising in the former cases may be due to my own lack of imagination rather than a broader lack of understanding. This approach has some dangers, but I hope that it will highlight interesting questions for future research.

It is probably not useful to over-define the concept of robust chaos. Roughly speaking a family of dynamical systems has robust chaos if the existence of a chaotic

\footnotetext{
a e-mail: p.a.glendinning@manchester.ac.uk
} 
attractor is persistent as the parameters vary. In other words, a map in an $m$ parameter family of maps is said to have robust chaos if it has a chaotic attractor and all sufficiently small changes in the $m$ parameters yield maps with a chaotic attractor. Sometimes it is useful to add the condition that the chaotic attractor is the unique attractor of the system.

Of course, the question of how the parametrization is defined was not addressed and so the 'definition' above is liable to abuse. It is easy to define a family in which all members of the family are conjugate via a linear transformation, and hence the dynamics of every member of the family is equivalent. This is not in the spirit of $[3,4]$, though it has been used, and van Strien [21] addresses this point quite effectively.

Robust chaos was originally defined in the context of the border collision normal form [17]. Let $z=(x, y)^{T}$ then the border collision normal form, with the notation of $[4]$, is the continuous map

$$
z_{n+1}=F\left(z_{n}\right)= \begin{cases}J_{L} z_{n}+m & \text { if } x_{n}<0 \\ J_{R} z_{n}+m & \text { if } x_{n} \geq 0\end{cases}
$$

where

$$
J_{k}=\left(\begin{array}{cc}
\tau_{k} & 1 \\
-\delta_{k} & 0
\end{array}\right), \quad k=L, R, \quad \text { and } m=\left(\begin{array}{c}
\mu \\
0
\end{array}\right) .
$$

The parameters $\tau_{k}$ and $\delta_{k}$ are generally taken to be fixed, and $\mu$ is the bifurcation parameter. Following [4] we will concentrate on parameters with $\mu>0$ and hence without loss of generality $\mu=1$ (by rescaling the variables) and

$$
0<\delta_{k} \leq 1, \quad k=L, R, \quad \tau_{L}>1+\delta_{L}, \quad \tau_{R}<-\left(1+\delta_{R}\right) .
$$

We will denote the set of border collision normal forms (1), (2) with $\mu=1$ and (3) as $B C N F_{R C}$.

These maps arise naturally in the study of the local bifurcations of piecewise smooth maps [17]. Consider a general family of piecewise smooth maps of the plane and suppose that at some critical value of the parameter, $\mu=0$ say, the map has a fixed point on a switching surface. By a change of coordinates the switching surface can (generically) be transformed to $x=0$ and the fixed point to the origin. Continuity across the switching surface implies that the second columns of the Jacobian matrices of the map on each side of the switching surface evaluated at the fixed point are equal, and by a further linear transformation this can (generically again) be taken to be 1 and 0 as in (2). Assuming that the curve of fixed points intersects the switching surface transversally as the parameter is varied, the leading order constant term can be chosen to be $(\mu, 0)^{T}$ by rescaling the parameter and a further translation of the $y$-variable if necessary. The result is (1) if nonlinear terms of the general map are ignored. Since this is the generic description of the local behaviour when a fixed point runs into the switching surface (or border), this is called the border collision normal form.

If $F \in B C N F_{R C}$ then $F$ has two fixed points, $X$ in $x>0$ and $Y$ in $x<0$, with

$$
X=\left(\frac{1}{1+\delta_{R}-\tau_{R}},-\frac{\delta_{R}}{1+\delta_{R}-\tau_{R}}\right), \quad Y=\left(-\frac{1}{\tau_{L}-1-\delta_{L}}, \frac{\delta_{L}}{\tau_{L}-1-\delta_{L}}\right) .
$$

Both are saddles: the Jacobian matrix at $Y, J_{L}$, has one eigenvalue greater than one and the other (also positive) less than one whilst the Jacobian matrix at $X$, $J_{R}$, has one eigenvalue less than -1 and the other between -1 and zero. Note that since $\delta_{k}>0, k=L, R, F$ is a homeomorphism and the image of the half-plane with 
$x<0$ is the half-plane with $y>0$ whilst the image of the half-plane with $x>0$ is the half-plane with $y<0$. Note also that the $x$-axis is the image of the $y$-axis. These observations will be useful later.

Banerjee et al. $[3,4]$ use arguments based on the geometry of the stable and unstable manifolds of $X$ and $Y$ to suggest that border collision normal forms in $B C N F_{R C}$ have robust chaos. These arguments are discussed in Section 2, where we highlight some issues in the sketch proof presented in [4]. The issues do not imply that the statement is incorrect, but some of the arguments do appear to be incomplete (as is normal in a sketch proof). In Section 3 we indicate how Misiurewicz [16] resolves some of the issues raised if the determinants $\delta_{k}$ are negative. In Section 4 we describe Young's approach to the problem [24], and in Section 5 the application of this approach to the border collision normal form is discussed. In Sections 6 and 7 these ideas are applied to the negative determinant case of Misiurewicz and the classic parameters of (3) respectively, showing how the conditions of Young's Theorem can be verified numerically. Finally, in Section 8 recent results on robust chaos for expanding maps are given. In this case it is possible to have fully two-dimensional attractors for open sets of parameters, whilst those of the classic robust chaos are quasi-one-dimensional with a fractal structure in the second dimension.

This article concentrates on the questions arising from the important observations in [4]. There are many other approaches to piecewise smooth systems (e.g. [1]), and although, as sketched above, the border collision normal form was derived in the context of the bifurcations of piecewise smooth maps in 1992 [17], its simplicity of form and intriguing complexity of dynamics had already been recognised (e.g. [8,14]). A full historical discussion of the approaches to, and observations of, complicated dynamics for these maps is beyond the scope of this article. See [19] for a more detailed account.

It is worth emphasising that the close scrutiny of the sketch proof in [4] is worthwhile because of the importance of the idea of robust chaos in the study of piecewise smooth systems. In other words, whilst the discussion of Section 2 presents a critique of [4], it is not intended in any way to be critical of the fundamental insights of that paper.

\section{Geometry of the stable and unstable manifolds}

The analysis of [4] involves three steps. First the stable and unstable manifolds of $Y$ (the fixed point in $x<0$ ) are used to define a trapping region, i.e. a polygon such that all initial conditions starting within the polygon remain in the polygon for all time. This implies that there is at least one attractor in the polygon. Second, it is shown that there is a transverse intersection between the stable and unstable manifolds of $X$ (the fixed point in $x>0$ ), and hence that there is 'a chaotic orbit' [4]. Finally, these two observations together with the existence of a heteroclinic connection between the unstable manifold of $Y$ and the stable manifold of $X$ are used to show that the "chaotic orbit' is the unique attractor in the polygon. Banerjee et al. [4] also use the (stated) continuity of Lyapunov exponents to argue that the attractor is robust, though this is unnecessary since if the previous three steps are correct then there is a unique chaotic attractor at each parameter value identified and no further work is required to prove that the attractor is robust.

To show that there must be some issues about the proof consider the attractor of Figure 1, which is a numerical extension of the example in [5] but with nonzero values of the determinants, a similar idea can be found in [15]. The parameters satisfy the conditions of the statements from [4], but in Figure 1b the attractor has two components which are bounded away from the fixed point $X$ which lies between 

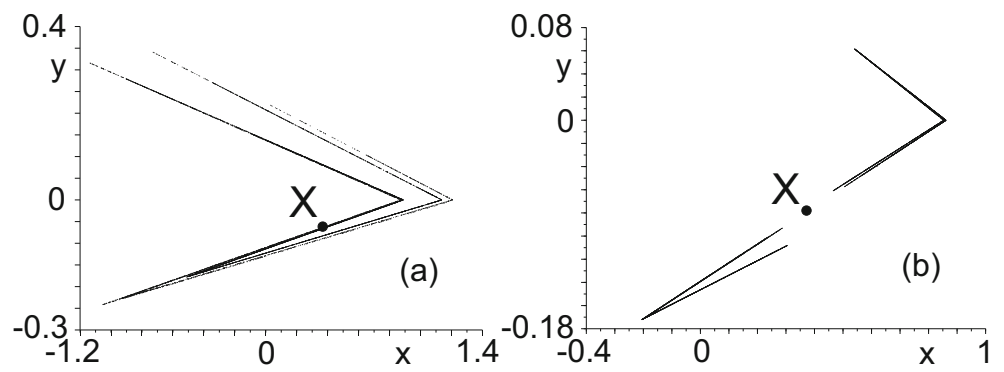

Fig. 1. 10000 iterates of the initial condition $(0.2,-0,1)$ of the border collision normal form showing the fixed point $X$ and strange attractors with one and two components respectively. (a) $\tau_{L}=1.8, \tau_{R}=-1.7, \delta_{L}=0.3, \delta_{R}=0.2$, so $X \approx(0.3448,-0.0690)$. For these values of the parameter the left hand side of (5) is approximately 0.1865 , so Lemma 1 holds, and the left hand side of (9) is approximately 1.6757 so Lemma 2 also holds. (b) $\tau_{L}=1.4$, $\tau_{R}=-1.4, \delta_{L}=0.3, \delta_{R}=0.2$, so $X \approx(0.3846,-0.0769)$. For these values of the parameter the left hand side of (5) is approximately 0.0582 , so Lemma 1 holds, and the left hand side of (9) is approximately -0.1140 so Lemma 2 does not hold.

the two components. Assuming that the chaotic orbit referred to in [4] is a chaotic set associated with the transverse homoclinic orbit to $X$, then $X$ is in the closure of the chaotic set and hence in the attractor. This is clearly not the case here, so some element of the sketch proof will need modification. For a more detailed description of the homoclinic bifurcations in piecewise-smooth systems see $[15,20,22]$.

Lemma 1. Suppose that $F \in B C N F_{R C}$ and denote the eigenvalues of $J_{L}$ by $\lambda_{k L}$, $k=1,2$ with $0<\lambda_{2 L}<1<\lambda_{1 L}$. If

$$
\tau_{L} \delta_{L}-\delta_{L}^{2}-\delta_{L} \lambda_{2 L}-\delta_{L} \delta_{R}+\tau_{R} \delta_{L} \lambda_{1 L}+\delta_{R} \lambda_{2 L}-\tau_{R} \delta_{L}>0
$$

then there exists a closed polygonal region $\mathcal{D}$ such that $z \in \mathcal{D}$ implies $F(z) \in \mathcal{D}$.

Note that (5) is precisely equation (5) of [4]. Of course, the fact that $z \in \mathcal{D}$ implies $F(z) \in \mathcal{D}$ means that $F^{n}(z) \in \mathcal{D}$ for all $n=1,2,3, \ldots$.

Proof of Lemma 1: Let $X=\left(X_{1}, X_{2}\right)^{T}$ and similarly for other points. The geometry of the stable and unstable manifolds of $Y$ is shown in Figure 2a. The eigenvalues $\lambda_{k L}$ are the solutions of $\lambda^{2}-\tau_{L} \lambda+\delta_{L}=0$ and the corresponding eigenvectors are $\left(-\lambda_{k L}, \delta_{L}\right)^{T}$. Using this it is straightforward to calculate the intersection $D$ of the local unstable manifold of $Y$ with the $x$-axis and the intersection $S$ of the local stable manifold of $Y$ with the $y$-axis:

$$
D=\left(\frac{\lambda_{1 L}-1}{\tau_{L}-1-\delta_{L}}, 0\right)^{T} \quad S=\left(0,-\frac{\lambda_{1 L}-\delta_{L}}{\tau_{L}-1-\delta_{L}}\right)^{T} .
$$

It is straightforward to show that (3) implies that $D_{1}>1$. The stable manifold of $Y$ bends on the $y$-axis and the continuation of the branch $Y S$ intersect the $x$-axis at $C$ where

$$
C=\left(\frac{\delta_{L}\left(\tau_{L}-\delta_{L}-\lambda_{2 L}\right)}{\left(\tau_{L}-1-\delta_{L}\right)\left(\delta_{R} \lambda_{2 L}-\tau_{R} \delta_{L}\right)}, 0\right)^{T} .
$$

Now suppose that $D_{1} \leq C_{1}$, and so $1<D_{1}<C_{1}$. Then the $x$-axis with $0<x<C_{1}$ maps to the line segment $\mathcal{L}$ connecting $(1,0)^{T}$ to $F(C)$ and this line contains $F(1,0)=$ $\left(\tau_{R}+1,-\delta_{R}\right)^{T}$. Since $\tau_{R}<-1$ this lies in $x<0$ and so the 'rest' of the line $\mathcal{L}$ does 

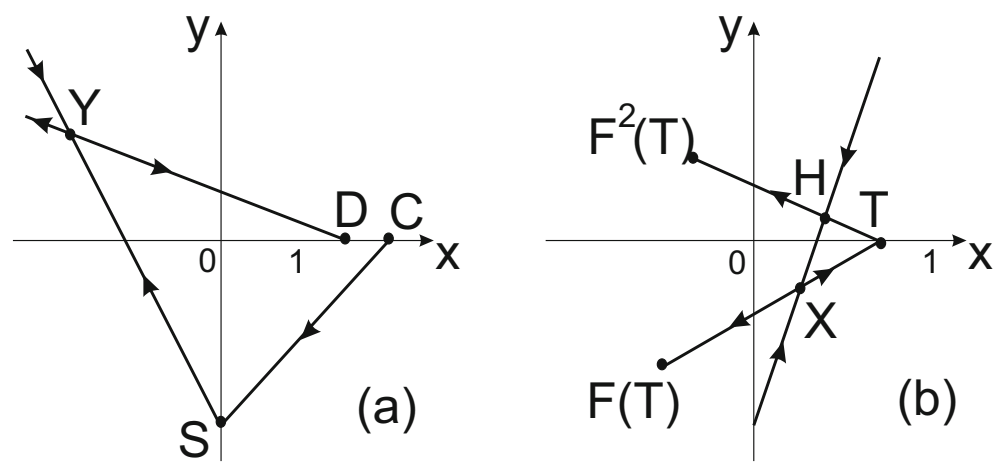

Fig. 2. Geometry of the invariant manifolds. (a) For the fixed point $Y$; (b) for the fixed point $X$ showing a homoclinic point, $H$.

too, in particular $F(C)$ lies in $x<0$. (This is important - it is necessary to ensure that the point $C$ is actually on the stable manifold of $Y$ and not simply on the extension of this line.) Since $F(C)$ is by definition on the line $Y S$, this also shows that $F(D)$ is on the line segment connecting $F(C)$ and $(1,0)^{T}$.

Now consider the region $\mathcal{D}=Y D C S$. By construction the image of the boundary of $\mathcal{D}$ is in $\mathcal{D}$ and hence $F(\mathcal{D}) \subseteq \mathcal{D}$.

Now consider the geometry of the stable and unstable manifolds of $X$ (see Fig. 2b). The eigenvalues of $J_{R}$ are $\lambda_{k R}, k=1,2$ satisfying $\lambda^{2}-\tau_{R} \lambda+\delta_{R}=0$ and (3) implies that they can be labelled so that $\lambda_{1 R}<-1<\lambda_{2 R}<0$. Both the local stable and unstable manifolds have positive slopes, the steeper being the stable manifold. The local unstable manifold is the line segment $T F(T)$ where $T$ is the intersection of the unstable manifold with the $x$-axis:

$$
T=\left(\frac{1-\lambda_{1 R}}{1+\delta_{R}-\tau_{R}}, 0\right)
$$

(remember $\tau_{R}<-1$ ). A short algebraic manipulation shows that $0<T_{1}<1$. The extension of the unstable manifold from $T$ into $y>0$ will be the image of the line segment $F(T) F^{-1}(T)$, i.e. the image of the part of the local unstable manifold that is in $x<0$. This is a line segment $F^{2}(T) T$ and since $F(T)$ is in $x<0$ then $F^{2}(T)$ is in $y>0$ as shown. Thus there will be a homoclinic intersection between the local stable manifold of $X$ and this extension of the unstable manifold of $X$ provided $F^{2}(T)$ lies to the left of the local stable manifold as indicated in Figure $2 \mathrm{~b}$ resulting in the homoclinic intersection point $H$.

Lemma 2. Suppose that $F \in B C N F_{R C}$ and denote the eigenvalues of $J_{R}$ by $\lambda_{k R}$, $k=1,2$, with $\lambda_{1 R}<-1<\lambda_{2 R}<0$. If

$$
\left(\tau_{L} \tau_{R}-\delta_{R}\right) \lambda_{1 R}+\left(\frac{\delta_{L}}{\delta_{R}}+\delta_{L}-1\right) \lambda_{2 R}-\tau_{R} \delta_{L}-\tau_{L} \delta_{R}+\tau_{R}-\tau_{L}>0,
$$

then the stable and unstable manifolds of $X$ intersect transversely.

Proof: By brute force calculation $F^{2}(T)$ lies on the stable manifold of $X$ if the left hand side of $(9)$ is zero.

I am not aware of the condition (9) in the literature, although as we shall see it explains the apparently anomalous behaviour of Figure 1. To see that (9) is not 
a consequence of the existence of the bounding region $\mathcal{D}$ it is easier to consider the special case

$$
\delta_{L}=\delta_{R}=b, \quad \tau_{L}=-\tau_{R}=a .
$$

In this case (5) becomes

$$
2 a-2 b-\lambda_{2 L}-a \lambda_{1 L}+\lambda_{2 L}>0
$$

and as $b \rightarrow 0, \lambda_{1 L} \rightarrow a$ and $\lambda_{2 L} \rightarrow 0$ so the condition is simply $2-a>0$ or $a<2$. Similarly (9) becomes

$$
-2 a(1-b)+b \lambda_{2 R}-\left(a^{2}+b\right) \lambda_{1 R}>0
$$

and as $b \rightarrow 0, \lambda_{1 R} \rightarrow-a$ and $\lambda_{2 R} \rightarrow 0$ so the condition is simply $-2 a+a^{3}>0$ or $a>\sqrt{2}$. This shows that if (5) holds then for some parameters (9) holds whilst for others it does not. Moreover, the thresholds in the limit $b \rightarrow 0$ make a connection with one-dimensional tent maps (unimodal maps $x \rightarrow 1-a|x|$ with slope of modulus $a, 1<a \leq 2$ ) blindingly obvious - though I confess I did not think of it until I had gone through this analysis. If $\sqrt{2}<a \leq 2$ then the attractor of the tent map is an interval and the non-trivial fixed point (the fixed point on the side that the map has negative slope) has a homoclinic connection, whilst if $1<s<\sqrt{2}$ there is no homoclinic connection to the non-trivial fixed point and the attractor is contained in the union of two disjoint intervals. This is essentially the explanation for Figure 1: there is no homoclinic connection to $X$, though presumably there is a transverse homoclinic orbit to an unstable orbit of period two since the attractor appears to have two components.

Having clarified the conditions for the existence of a transverse homoclinic intersection between the stable and unstable manifolds of $X$, the next step is to argue that there is a unique chaotic attractor assuming that (5) and (9) are satisfied. Note that if (9) is not satisfied then there can still be robust chaos involving homoclinic connections to orbits other than the fixed point $X$, but we will concentrate here on the argument of [4].

There are two natural conjectures arising from the discussion above.

Conjecture 3 The set of $F \in B C N F_{R C}$ such that (5) holds has robust chaos.

This is the original statement of [4] and [3]. Lemma 2 shows that the argument of [4] based on transverse intersections of the stable and unstable manifolds of $X$ do not hold here, although it is quite possible that by replacing $F$ by $F^{2^{k}}$ for some $k \geq 1$ in different regions of the parameter space there might be a way to extend their argument over the whole of this parameter space. Indeed, my guess is that this conjecture is true; all I am pointing out is that the received argument is not sufficient to establish it.

Conjecture 4 The set of $F \in B C N F_{R C}$ such that (5) and (9) hold has robust chaos.

For the parameter values of Conjecture 4 there is a trapping region and hence at least one attractor (Lemma 1) and a transverse intersection between the stable and unstable manifolds of $X$ (Lemma 2), and so the geometry is as described in [4].

The argument of [4] involves an application (uncontroversial) of the Lambda Lemma $[2,18]$. Putting the two parts of Figure 2 together we see that there is a transverse intersection between the local unstable manifold of $Y$ and the local stable manifold of $X$, at a heteroclinic point $T$ say. By definition $F^{k}(T) \rightarrow X$ and $F^{k}(T)$ lies in $x>0$ for all $k>0$. Hence given any $\eta>0$ there is a small part of the unstable 
manifold of $Y$ through $T$ which is within $\eta$ of the local unstable manifold of $X$ after some number of iterations in $x>0$. Let $W^{u}(X)$ denote the unstable manifold of $X$. Since the unstable manifold of $X$ is the union of images of any sufficiently small part of the local unstable manifold of $X$ containing $X$, the continuity of $F$ implies that given any point $x \in W^{u}(X)$ and $\epsilon>0$ then by choosing $\eta$ small enough there will be a point on the image of the unstable manifold of $Y$ close to $T$ that maps within $\epsilon$ of $x$. Hence for all $x \in W^{u}(X)$ and $\epsilon>0$ there exists $y \in W^{u}(Y)$ such that $|x-y|<\epsilon$.

Banerjee et al. now argue [4] that this, together with the observations made above, is enough to establish Conjecture 4. Their (sketch) demonstration, with the notation above, is [4]: Since $W^{u}(Y)$ comes arbitrarily close to $W^{u}(X)$, the attractor must span $W^{u}(Y)$ in one side of the heteroclinic point. Since all initial conditions in $x<0$ tend to $W^{u}(Y)$ and all initial conditions in $x>0$ converge to $W^{u}(X)$, and since there are points in $W^{u}(Y)$ in every neighbourhood of $W^{u}(X)$, we conclude that the attractor is unique. Precisely the same wording is used in the expanded version [3] so we learn nothing new there.

The first sentence is not easy to understand; it is possible that the following argument is intended. The chaotic set comes arbitrarily close to the unstable manifold of $X$ and hence, as the chaotic set is recurrent and $F$ is a homeomorphism, arbitrarily close to preimages of this set, and since at least one side of the unstable manifold of $Y$ through $T$ comes arbitrarily close to the local unstable manifold of $X$, then the attractor is arbitrarily close to (at least) one side of the unstable manifold of $Y$ in a neighbourhood of $T$. However, the use of the word span suggests something stronger, that every point on the unstable manifold of $X$ close to $T$ and on one side of $T$ is arbitrarily close to the attractor, and this is not clear from the description of the chaotic orbit.

The argument behind the second sentence is also unclear. It is true that under iteration in $x<0$ (resp. $x>0$ ) orbits tend to the local unstable manifold of $Y$ (resp. $X)$. However, this does not imply contraction towards an arbitrary point on the global unstable manifolds. Indeed, the separation of two points in $W^{u}(X)$ with $x<0$ (and $y<0$ ) that lie on a line segment parallel to the unstable manifold of $Y$ is increased by iteration in $x<0$. However, points separated by a vector in the stable eigendirections (e.g. the eigenvector of $\lambda_{2 L}$ in $x<0$ ) are contracted so provided such points exist locally, or exist sufficiently frequently locally, the an argument like this should hold.

The point of the previous two paragraphs is that although Banerjee et al $[3,4]$ have described an important and real phenomenon, it is worth challenging the community to work harder on the precise statements and arguments that justify their conclusions. This is not simply a mathematical nicety; I feel that this understanding would help understand other features of piecewise smooth systems too.

Establishing the status of the two conjectures above is left as an open problem (and I hope I am not being stupid for not seeing the full argument). Let me help (or muddy the waters) by adding another conjecture which makes the possible status of 'the chaotic orbit' of [4] more explicit. Reasons for believing this conjecture are given in the next section, Section 3.

Conjecture 5 For $F \in B C N F_{R C}$ there is an open set of parameter values such that (5) and (9) hold, the sytem has robust chaos, and the attractor is the closure of $W^{u}(X)$.

\section{Misiurewicz's Theorem and Lozi maps}

Lozi [14] introduced the maps

$$
L(x, y)=(1-a|x|+y, b x)
$$


as a piecewise linear model of the (smooth) Hénon map [12]. The hope was that the lack, for typical parameters, of a critical set on which the Jacobian has a zero eigenvalue would make it possible to prove the existence of strange attractors in this model problem, where the existence of strange attractors for the Hénon map was proving remarkably hard to establish rigorously. The Lozi map is a subclass of the border collision normal form with

$$
-\tau_{R}=\tau_{L}=a, \quad \delta_{L}=\delta_{R}=-b, \quad \mu=1 .
$$

If $b>0$ (i.e. $\delta_{L}$ and $\delta_{R}$ both equal and negative), $a>0$ and $a+1-b>0$ then there is a fixed point fixed point $X$ in $x>0$,

$$
X=\left(\frac{1}{a+1-b}, \frac{b}{a+1-b}\right)^{T} .
$$

This is a saddle, with eigenvalues $\lambda_{2 R}<-1<0<\lambda_{1 R}<1$ if

$$
a>1-b .
$$

In 1980 Misiurewicz showed that provided some further conditions hold there is indeed robust chaos, although his result pre-dates the definition of [4]. A subset $\mathcal{A}$ of $\mathbb{R}^{2}$ is topologically transitive if for all open $U_{k} k=0,1$ with $U_{k} \cap \mathcal{A} \neq \emptyset$ there exists $n$ such that $F^{n}\left(U_{0}\right) \cap U_{1} \neq \emptyset$.

Theorem 1. (Misiurewicz [16]) Suppose that

$$
a>0 \quad \text { and } \quad 0<b<\min \left\{4-2 a, a \sqrt{2}-2, \frac{a^{2}-1}{2 a+1}\right\} .
$$

Then the attractor of the Lozi map (11) is the closure of $W^{u}(X)$ and the map is topologically transitive on this set.

Note that (14) is implied by the conditions of the theorem. Parts of the proof are similar to those rehearsed in Section 2, but simplified reflecting the fact that the stable and unstable manifolds of a fixed point in $x<0$ are not needed. Thus Conjecture 5 holds in this negative determinant case. In the case of positive determinants considered in Section 2 the extra flipping due to the fact that the stable eigenvalue at $X$ is negative complicates the geometry. We leave it as a (second) challenge to modify Misiurewicz's construction to obtain a proof in a similar style.

\section{Invariant measures and Young's Theorem}

Very little has been written about the existence of invariant measures for the dynamics of the normal form within the border collision community. However, Lozi maps and their generalizations have been considered in this light $[7,13,24]$ and this provides a theoretical framework within which the existence of measures can be established. The key to this is Young's Theorem [24]. The existence of an invariant measure with non-trivial support shows that there is an attractor on which the dynamics acts as a sort of probability distribution asymptotically. We will not go into details here and the reader unfamiliar with invariant measures should simply think of this as a way of describing chaos (although not all invariant measures are chaotic). See [13] for a readable introduction.

Young [24] provides a result that can be used to prove the existence of chaotic attractors in a wide class of maps that include the border collision normal form. Let 
$R=[0,1] \times[0,1]$ and let $S=\left\{a_{1}, \ldots, a_{k}\right\} \times[0,1]$ be a set of vertical switching surfaces with $0<a_{1}<\cdots<a_{k}<1$. Then $f: R \rightarrow R$ is a Young map if $f$ is continuous, $f$ and its inverse are $C^{2}$ on $R \backslash S$ and $f=\left(f_{1}, f_{2}\right)^{T}$ satisfies the expansion properties (H1)-(H3) below on $R \backslash S$.

$$
\begin{array}{r}
(H 1) \quad \inf \left\{\left(\left|\frac{\partial f_{1}}{\partial x}\right|-\left|\frac{\partial f_{1}}{\partial y}\right|\right)-\left(\left|\frac{\partial f_{2}}{\partial x}\right|-\left|\frac{\partial f_{2}}{\partial y}\right|\right)\right\} \geq 0, \\
(H 2) \quad \inf \left(\left|\frac{\partial f_{1}}{\partial x}\right|-\left|\frac{\partial f_{1}}{\partial y}\right|\right)=u>1, \quad \text { and } \\
(H 3) \sup \left\{\left(\left|\frac{\partial f_{1}}{\partial y}\right|+\left|\frac{\partial f_{2}}{\partial y}\right|\right)\left(\left|\frac{\partial f_{1}}{\partial x}\right|-\left|\frac{\partial f_{1}}{\partial y}\right|\right)^{-2}\right\}<1 .
\end{array}
$$

Young's Theorem describes measures that project nicely onto one-dimensions. Technically this is expressed as having absolutely continuous conditional measures on unstable manifolds. Intuitively this means that locally the measure projects nicely onto one dimension.

Let $J a c(f)$ denote the Jacobian matrix of $f$ and recall that $u$ is defined in (H2).

Theorem 2. (Young [24]) If $f$ is a Young map, $|J a c(f)|<1$ for $x \in R \backslash S$, and there exists $N \geq 1$ such that $u^{N}>2$ and $f^{k}(S) \cap S=\emptyset, 1 \leq k \leq N$, then $f$ has an invariant probability measure that has absolutely continuous conditional measures on unstable manifolds.

Since the result is for piecewise $C^{2}$ maps and the conditions only depend on derivatives this result has the important corollary that results for the piecewise linear border collision normal form, which should more correctly be called a truncated normal form, persist when small nonlinear terms are added.

In the piecewise linear context, consider a general piecewise affine continuous map of the form (1) but with linear parts $J_{R}$ and $J_{L}$ defined by

$$
J_{k}=\left(\begin{array}{cc}
A_{k} & B \\
C_{k} & D
\end{array}\right),
$$

$k=R, L$ and with a general constant $m \in \mathbb{R}^{2}$. Then the assumptions (H1)-(H3) above become

$$
\begin{aligned}
\left(\left|A_{k}\right|-|B|\right)-\left(\left|C_{k}\right|-|D|\right) & \geq 0, & & k=R, L \\
\left|A_{k}\right|-|B| & >1, & & k=R, L \\
(|B|+|D|) /\left(\left|A_{k}\right|-|B|\right)^{2} & <1, & & k=R, L .
\end{aligned}
$$

Corollary 6 Let $F: \mathbb{R}^{2} \rightarrow \mathbb{R}^{2}$ be a map of the form (1) with arbitrary $m \in \mathbb{R}^{2}$ and matrices $J_{k}, k=R, L$ given by (16) with coefficients which satisfy (17). Suppose that the map takes some rectangle $D=[a, b] \times[c, d]$ with $a<0<b$ into itself and let $S=$ $\{0\} \times[c, d]$ (the segment of the $y$-axis in $D)$. Let $u=\min _{k=R, L}\left(\left|A_{k}\right|-|B|\right)$. If there exists an integer $N>0$ such that $u^{N}>2$ and $F^{p}(S) \cap S=\emptyset$ for $1 \leq p \leq N$ then $F$ has an attractor with an invariant probability measure that has absolutely continuous conditional measures on unstable manifolds.

$N$ describes how many iterates of the map are applied before returning to the critical set, and hence provides a bound on the horizontal expansion of segments. This implies that Young's Theorem can be slightly rewritten in a stronger form. 
Conjecture 7 (Modified Young's Theorem) Theorem 2 remains true if the final condition is replaced by 'there exists an integer $N \geq 1$ such that $u^{N}>2$ and $f^{p}(S) \cap S=$ $\emptyset$ for $1 \leq p<N$.

This conjecture implies a similar reformulation of Corollary 6 . The only difference between Young's Theorem (Theorem 2) and Conjecture 7 is that the inequality on $p$ is replaced by strict inequality. Note that Young herself remarks that if $N=1$ then the condition is empty (which is the case for the strict inequality of Conjecture 7 but not for the inequality of Theorem 2) and has an illustration with $f(S) \cap S \neq \emptyset$ claiming this is allowed, which is not the case if equality holds. This, together with the remark that the importance of $N$ is that the proof of the theorem can be repeated with $f^{N}$ instead of $f$ in this case, strongly suggests that the less than or equals sign is a typographical error and that Conjecture 7 is correct, though I do not consider myself a strong enough ergodic theorist to state this categorically ${ }^{1}$. However, all the numerical results below assume this modified result. If it is not correct then the regions identified need modification but the general structure of the numerical programmes is unaltered.

For the border collision normal form where the matrices $J_{R}$ and $J_{L}$ are

$$
\left(\begin{array}{cc}
\tau_{k} & 1 \\
-\delta_{k} & 0
\end{array}\right), \quad \alpha=L, R
$$

see (2), so the conditions (17) become

$$
\begin{array}{rlrl}
\left|\tau_{k}\right|-1-\left|\delta_{k}\right| & \geq 0, & k=R, L \\
\left|\tau_{k}\right| & >2, & k & =R, L \\
1 /\left(\left|\tau_{k}\right|-1\right)^{2} & <1, & k & =R, L .
\end{array}
$$

The third of these equations is implied by the second, so only the first two of these equations act as conditions. Unfortunately the second condition is restrictive in a way which means that (for example) the Lozi map with parameters defined by (15) cannot be considered directly because $a$ (which is effectively $\tau_{k}$ ) is less than two. But, as Young herself points out, this problem can be avoided by a simple scaling. Two other problems need to be addressed before the result can be applied: the rectangular region $R$ must be identified and then the exponent $N$ computed. The first of these problems needs some thought, and it is easier to use a non-rectangular region with a view to minimizing $N$ (the proof of Theorem 2 is unaffected provided the switching surfaces have slopes with modulus greater than one). The problem of determining $N$ is where computer simulations come into their own. These three factors: scaling, the existence of a trapping region and the calculation of the exponent $N$ are the subject of the next section.

\section{Young's Theorem and the border collision normal form}

Given $\varepsilon>0$ let $y=\varepsilon z$, then in terms of the new coordinates $(x, z)$ the border collision normal form $F \in B C N F_{R C}$ is

$$
\left(\begin{array}{l}
x_{n+1} \\
z_{n+1}
\end{array}\right)=\left(\begin{array}{cc}
\tau_{k} & \varepsilon \\
-\delta_{k} / \varepsilon & 0
\end{array}\right)\left(\begin{array}{l}
x_{n} \\
z_{n}
\end{array}\right)+\left(\begin{array}{l}
1 \\
0
\end{array}\right)
$$

\footnotetext{
${ }^{1}$ I have also had informal confirmation of this from a researcher working directly in this area.
} 
with $k=R$ if $x_{n} \geq 0$ and $k=L$ if $x_{n} \leq 0$. Young's conditions on the derivatives, (17), therefore become

$$
\begin{aligned}
\varepsilon\left(\left|\tau_{k}\right|-\varepsilon\right)-\left|\delta_{k}\right| \geq 0, & & k=R, L \\
\left|\tau_{k}\right|-\varepsilon>1, & & k=R, L \\
\varepsilon /\left(\left|\tau_{k}\right|-\varepsilon\right)^{2}<1, & k & =R, L .
\end{aligned}
$$

If $\varepsilon \in(0,1)$ and $\left|\delta_{k}\right|<\varepsilon$ then the second inequality, $\left|\tau_{k}\right|>1+\varepsilon$, implies both the first and the third.

Suppose $\mathcal{D}$ is a trapping region for the border collision normal form in the standard coordinates (1), and $\mathcal{D}^{\prime}$ is the corresponding region in the new coordinates $(x, z)$. Then clearly $\mathcal{D}^{\prime}$ is a trapping region, and the intersection, $\mathcal{C}$, of $\mathcal{D}$ with the critical line $x=0$ is mapped by the coordinate transformation to the intersection, $\mathcal{C}^{\prime}$, of the transformed critical line ( $\operatorname{still} x=0)$ with $\mathcal{D}^{\prime}$. Since the map $(x, y) \rightarrow(x, z)$ is a differentiable conjugacy for the dynamics if $\varepsilon \neq 0$, the geometric condition of Young's Theorem can either be written in the old coordinates or the new coordinates, and we will choose to continue to work in the new coordinates.

In the statement of Young's Theorem the trapping region is a rectangle, but the proof relies only on the expansion properties of near-horizontal segments, and so works for any invariant region which intersects the critical line and its images nicely. In particular we may take a convex trapping region that intersects vertical lines in at most one connected component instead of $R$. These comments lead to the following reformulation of Young's Theorem for the border collision normal form.

Theorem 3. Let $F: \mathbb{R}^{2} \rightarrow \mathbb{R}^{2}$ be the border collision normal form (1) and suppose $F$ has a convex trapping region $\mathcal{D}$ which intersects the critical line $\{x=0\}$ in a closed, non-empty line segment $\mathcal{C}$. If Conjecture 7 holds and there exists $\varepsilon>0$ and $N \geq 1$ such that inequalities (20) hold, and $\left(\left|\tau_{k}\right|-\varepsilon\right)^{N}>2$ with $F^{p}(\mathcal{C}) \cap \mathcal{C}=\emptyset$ for $1 \leq p<N$, then $F$ has an attractor with an invariant probability measure that has absolutely continuous conditional measures on unstable manifolds.

As before, note that if $N=1$ then we interpret the final condition to be automatically true (as a property of the empty set) and the condition becomes simply that inequalities (20) hold and $\left|\tau_{k}\right|-\varepsilon>2$. We shall use the conditions from Theorem 3 to verify the existence of invariant measures for the border collision normal form.

Corollary 8 Suppose that $F \in B C N F_{R C}, F$ has a trapping region $\mathcal{D}$ which is mapped strictly inside itself, and Conjecture 7 holds for $F$. Let $v_{\varepsilon}$ be a continuous function on $\mathcal{D}$ such that $F+v_{\varepsilon}$ and its inverse are $C^{2}$ on $\mathcal{D} \backslash \mathcal{C}$, and $v$ and its first derivatives have modulus less than $\varepsilon$ on $\mathcal{D} \backslash \mathcal{C}$. Then for all $\epsilon>0$ sufficiently small, $F+v_{\varepsilon}$ has an attractor with an invariant probability measure that has absolutely continuous conditional measures on unstable manifolds.

Proof: For sufficiently small $\varepsilon>0, \mathcal{D}$ is a trapping region for $F+v$ since $F(\mathcal{D})$ is strictly contained in $\mathcal{D}$. Similarly, the assumptions of Young's Theorem hold for $F+v$ hold if $\epsilon$ is sufficiently small by the continuity of $F+v$ and its first derivatives in $\mathcal{D} \backslash \mathcal{C}$.

\section{The Lozi map revisited}

Detailed analysis of the geometry of invariant sets in these piecewise linear examples frequently flounders on the rocks of infeasibly long algebraic expressions and their interpretation. It is however often possible to express conditions implicitly in terms that a computer can verify using only the four elementary operations of addition, 

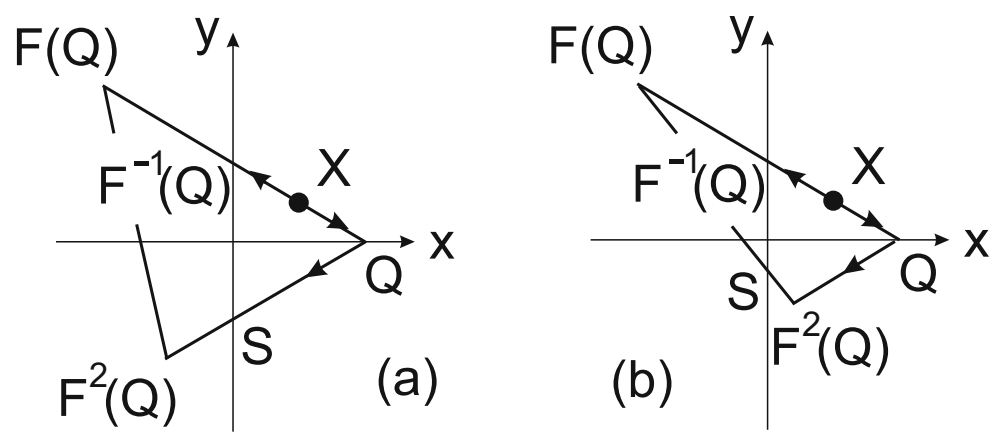

Fig. 3. Geometry of the trapping region for parameters which satisfy (15) of Theorem 1. (a) $F^{2}(Q)$ to the left of the $y$-axis; (b) $F^{2}(Q)$ to the right of the $y$-axis. $F^{-1}(Q)$ is the intersection of the line segment $Q F(Q)$ with the $y$-axis.

subtraction, multiplication and division. In this section we will combine the theoretical approach of the previous section with, where appropriate, numerical confirmation of the algebraic conditions that apply. To emphasise that this approach has been taken we add the caveat (Numerical) to all the theorems stated, and in the proofs the places where computer verification has been used is indicated by italics.

Our first result in this section applies Theorem 3 to the region of parameters identified in Misiurewicz's Theorem (Theorem 1).

Theorem 4. (Numerical) If Conjecture 7 holds then the attractor of the Lozi map described in Theorem 1 has an invariant probability measure that has absolutely continuous conditional measures on unstable manifolds.

Proof: Let $Q$ be the intersection of the unstable manifold of the fixed point $X$ with the $x$-axis. Then for parameters satisfying $(15) F(Q)$ is in $x<0$ and the triangle $Q F(Q) F^{2}(Q)$ is a trapping region [16]. The point $F^{2}(Q)$ may be in $x<0$ or $x>0$; the geometry is sketched in Figure 3.

The intersection of this trapping triangle with the critical line (the $y$-axis) is a vertical line segment $S F^{-1}(Q)$, where $S$ is the intersection of $F^{2}(Q) Q$ with the $y$-axis if $F^{2}(Q)$ is in $x<0$ as shown in Figure 3a, or the intersection of $F(Q) F^{2}(Q)$ with the $y$-axis if $F^{2}(Q)$ is in $x>0$ as in Figure 3b. If $F^{2}(Q)$ is on the $y$-axis then $S=F^{2}(Z)$.

To apply Young's Theorem in the form of Theorem 3 let $\varepsilon=b$ in (19). Then the three inequalities (20) are all satisfied provided

$$
a>1+b
$$

since $0<b<1$. It is easy to prove analytically that (21) holds for all parameters in (15) and that in fact $a-b>v$ where

$$
v=14-9 \sqrt{2} \approx 1.272 .
$$

(This is derived by considering $a-b$ at the intersection the lines $b=4-2 a$ and $b=\sqrt{2} a-2$, which lies just outside the region defined by (15) and gives a lower value for $a-b$ than any other point.) By direct calculation $v^{2}<1$ but $v^{3} \approx 2.058>2$.

Thus the line segment $F^{-1}(Q) S=\mathcal{C}$ represents the critical line in the trapping triangle, so we can apply Theorem 3 with $N=3$ and $u=v$ provided $F(\mathcal{C})$ and $F^{2}(\mathcal{C})$ are both disjoint from $\mathcal{C}$. Now, $F(\mathcal{C})=Q F(S)$ which is in $x>0$, and $F^{2}(\mathcal{C})=F(Q) F^{2}(S)$ which lies in $x<0$ provided $F^{2}(S)$ lies in $x<0$. The condition that $F^{2}(S)_{1}<0$ can 


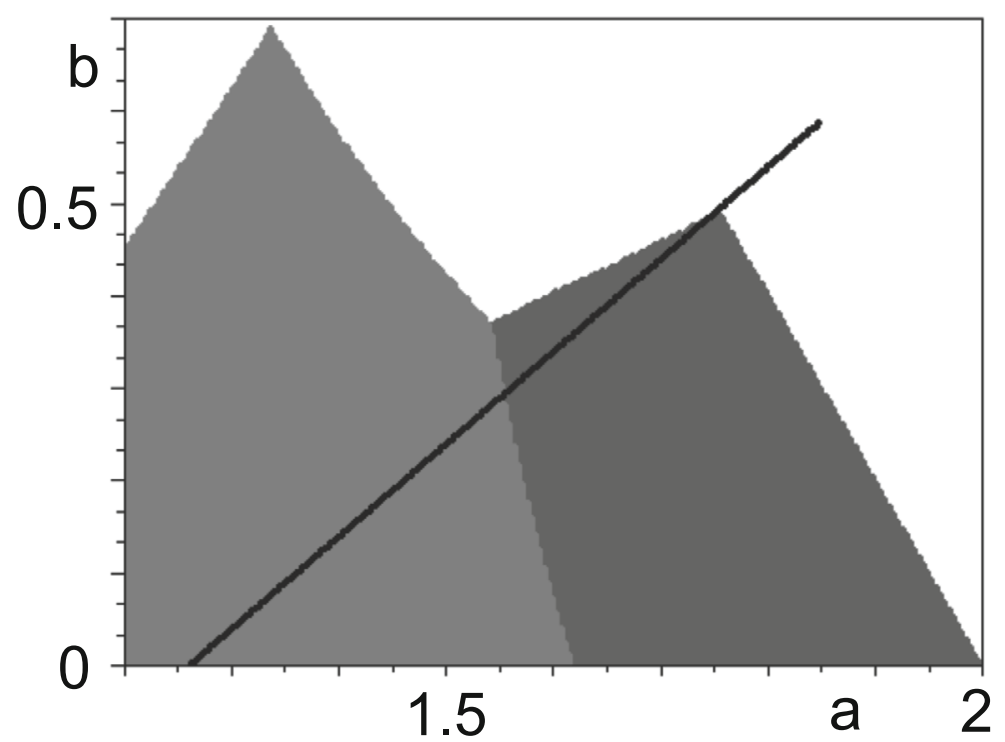

Fig. 4. Parameter space for the Lozi map: there is an attractor with a 'nice' invariant measure for parameters in the shaded region which lies below the line $b=a-2^{\frac{1}{3}}$ indicated in bold. This line leaves the shaded region just to the left of the local maximum in $b$. The right hand shaded region has an invariant region with $F^{2}(Q)$ to the right of the $y$-axis, the left hand region has an invariant region with $F^{2}(Q)$ on the left of the $y$-axis.

easily (but painfully) be calculated and yields a polynomial inequality, but we have checked numerically that $F^{2}(S)$ is in $x<0$ for all parameters defined by (15) and hence Theorem 3 can be applied to show the existence of an invariant measure as stated.

The numerical verification referred to in this proof is neither sophisticated nor exhaustive: a $100 \times 100$ grid was set up in parameter space containing the region defined by (15) and the position of $F^{2}(S)$ was calculated on this grid, checking that $F^{2}(S)_{1}<0$. Much more sophisticated approaches could clearly be used, but the importance of the result does not seem to merit that degree of effort!

It is also possible to consider parameter values outside the region considered by Misiurewicz (see Theorem 1). The triangle $Q F(Q) F^{2}(Q)$ described above is a trapping region over a much larger range of parameter values than the set defined by (15) in Theorem 1. Indeed, the results of Young [24] show the existence of a 'nice' invariant measure for all parameters in the shaded region of Figure 4 which lie below the line $(a-b)^{3}=2$. This, and the significance of the different shading in Figure 4, are explained below.

Theorem 5. Suppose that a Lozi map has a convex trapping region $\mathcal{D}$ which intersects the critical line $x=0$ on a line segment $\mathcal{C}$. If Conjecture 7 holds, $0<b<1$, and there exists $N>0$ such that

$$
(a-b)^{N}>2
$$

and $F^{k}(\mathcal{C}) \cap \mathcal{C}=\emptyset, 1 \leq k<N$, then $F$ has an attractor in $\mathcal{D}$ with an invariant probability measure that has absolutely continuous conditional measures on unstable manifolds. 
Proof: If $(a-b)^{N}>2$ then $a-b>1$ and so the inequalities (20) are satisfied with $\varepsilon=b<1$. This, together with (23) and the self-intersection condition for $\mathcal{C}$ imply that the conditions of Theorem 3 are satisfied and the result follows.

Corollary 9 Suppose that $a>1+b, 0<b<1$ and define $S$ and $Q$ as in the proof of Theorem 4. If Conjecture 7 holds and $Q F(Q) F^{2}(Q)$ is a trapping region, $(a-b)^{3}>2, F(S)$ lies in $x>0$ and both $F^{2}(S)$ lies in $x<0$, then $F$ has an invariant probability measure that has absolutely continuous conditional measures on unstable manifolds.

Proof: Since $\mathcal{C}$, the critical line in the trapping region, is the line segment $F^{-1}(Q) S$, $F(\mathcal{C})=Q F(S)$ and since $Q$ is in $x>0, F(\mathcal{C})$ is in $x>0$ provided $F(S)$ is in $x>0$. Similarly, as $F^{2}(\mathcal{C})=F(Q) F^{2}(S)$ and $F(Q)$ is in $x<0$, the interval $F^{2}(\mathcal{C})$ lies in $x<0$ if $F^{2}(S)$ is also in $x<0$.

Regions on which the assumptions of Theorem 9 hold are easy to calculate numerically and an example is shown in Figure 4 . Note that $(a-b)^{3}>2$ is the same as $b<a-2^{\frac{1}{3}}$; the straight line in Figure 4 is the boundary of this region. The right hand boundary of the shaded region below the line $b=a-2^{\frac{1}{3}}$ is the line $2 a+b=4$ as near as can be judged numerically. This is part of the boundary of the region defined by Misiurewicz [16], see Theorem 1. The line $b=a-2^{\frac{1}{3}}$ is an improvement on Misiurewicz, who uses the line $b=a \sqrt{2}-2$.

The issue to be determined numerically is whether $Q F(Q) F^{2}(Q)$ is a trapping region. Since the map is piecewise linear in each half-plane this is a simple question about whether the images of the points $Q, F(Q), F^{-1}(Q), F^{2}(Q)$ and $S$. Since the images of the first three of these points are known and in the closed triangle $Q F(Q) F^{2}(Q)$ only the images of the latter two points need to be checked. In the left hand shaded region $Q F(Q) F^{2}(Q)$ is a trapping region with $F^{2}(Q)$ in $x>0$ with the conditions on $F(S)$ and $F^{2}(S)$ satisfied, and the right hand shaded region is the same but with $F^{2}(Q)$ in $x<0$.

Thus Figure 4 is effectively itself a theorem. The structure of the computer programme used to generate it is as follows. For each parameter $(a, b)$ covering a given region of parameter space (e.g. in Figure 4 a $200 \times 200$ grid with $1.2<a \leq 2$ and $0<b \leq 0.8$ was used).

- Calculate $Q$ from its theoretical value, then $F(Q), F^{2}(Q)$ and $F^{3}(Q)$ by iteration.

- Calculate the points $U$ and $V$ on the (extended) lines $F(Q) F^{2}(Q)$ and $F^{2}(Q) Q$ respectively, which have $y$-coordinates equal to $\left[F^{3}(Q)\right]_{2}$.

- If $F^{3}(Q)_{1}<U_{1}$ and/or $F^{3}(Q)_{1}>V_{1}$ then go to the next value of $(a, b)$ as the triangle $Q F(Q) F^{2}(Q)$ is not a trapping region. Otherwise:

- If $F^{2}(Q)_{1}<0$ then calculate the point $S$ on the intersection of $F^{2}(Q) Q$ with the $y$-axis.

- If $F^{2}(Q)_{1}>0$ then calculate the point $S$ on the intersection of $F^{2}(Q) F(Q)$ with the $y$-axis.

- Calculate the iterates $F(S)$ and $F^{2}(S)$.

- If $F(S)_{1}>0$ and $F^{2}(S)_{1}<0$ then plot the point $(a, b)$ as these are on the same side of the $y$-axis as $F(O)$ and $F^{2}(O)$ respectively, and hence the first two images of the critical line $O S$ do not intersect $O S$.

- Repeat for the next value of $(a, b)$.

Attention has been restricted here to the Lozi map studied by Misiurewicz [16] described in Section 3, but analogous statements hold for the more general border collision normal form with negative determinants, $\delta_{k}<0$. 


\section{Attractors for robust chaos}

Although Lemma 1 provides a trapping region for the border collision normal form, this region is by no means optimal in general, and to maximize the value of $N$ in Young's Theorem (Theorem 2) it helps to minimize the size of the critical line that needs to be considered.

Recall the geometry of the stable and unstable manifolds of $Y$ shown in Figure 2a used to construct the trapping region of Lemma 1. The local unstable manifold of $Y$ has a fold on the $x$-axis at the point $D$ and (if $F \in B C N F_{R C}$ ) $D_{1}>1$ and so (as $\left.\tau_{R}<-1\right), F(D)$ lies in $x<0$ with $y<0$. Let $U$ be the intersection of the line $D F(D)$ with the (negative) $y$-axis, and let $V$ be the intersection of the extension of this line with the local stable manifold of $Y$, so $V$ lies to the left of the switching surface in $y<0$.

Lemma 10. If $F \in B C N F_{R C}$ satisfies (5) the closed triangle $Y D V$ is a trapping region.

Proof: Note that $F(D)$ lies to the right of $V$ (inside the original trapping region, which exists by Lemma 1$), Y$ is a saddle and $J_{L}$ has positive eigenvalues. The triangle $Y D V$ is the union of two parts, $Y O U V$ in $x<0$ and $O D U$ in $x>0$, where $O$ represents the origin.

Consider the image of the region $Y O U V . F(U)$ lies on the $x$-axis to the left of $D$ and the right of the local stable manifold of $Y, F(O)=(1,0)^{T}$ which is to the left of $D$ as well, and $F(V)$ is on the line $V Y$ as it is on the local stable manifold of $Y$. Thus the images of $Y, O, U$ and $V$ lie in $Y D V$ and so $F(Y O U V) \subseteq Y D V$.

Now consider the image of $O D U$. The previous paragraph establishes that $F(O)$ and $F(U)$ are in $Y D V$ and $F(D)$ is in $O D V$ by definition as it is on the line $D V$. Hence, as $F$ is affine in $x \geq 0 F(O D V) \subset Y D V$.

Theorem 6. Suppose $F \in B C N F_{R C}$ and (5) holds, and let $\varepsilon=\max \left\{\delta_{R}, \delta_{L}\right\}$. If there exists $N \geq 1$ such that

$$
\left(\left|\tau_{k}\right|-\varepsilon\right)^{N}>2, \quad k=R, L,
$$

both $F^{k}(O)$ and $F^{k}(U)$ lie on the same side of the critical line $x=0$ for $1 \leq k<N$, and Conjecture 7 holds, then $F$ has an attractor with an invariant probability measure that has absolutely continuous conditional measures on unstable manifolds.

Proof: By Lemma 10, YDV is a trapping region and we can restrict attention to this set.

Suppose that $\delta_{L} \geq \delta_{R}$. Let $\varepsilon=\delta_{L}$ and consider the change of variable in Section 5 which leads to (19). Equation (24) implies that $\left|\tau_{k}\right|-\varepsilon>1, k=R, L$, and so the second of inequalities (20) is satisfied. Since $\varepsilon<1$ the first and third inequalities of (20) are automatically satisfied. Hence the inequalities (20) hold and the theorem follows from the statement of Theorem 3. The argument is entirely analogous if $\delta_{R}>\delta_{L}$

Figure 5 shows the numerically computed regions where the conditions of this Theorem apply for $N=3,4,5,6$ (the regions exist for decreasing $\tau_{R}$ ). The structure of the computer programme used to generate this figure is essentially the same as the programme described at the end of Section 6 . Where more than one value of $N$ can be chosen we shade it in keeping with the lower value of $N$. The right hand boundary corresponds to equality in (5). Note that for larger $\left|\tau_{R}\right|$ our application of Young's Theorem does not even cover the whole of this boundary in the case $\delta_{R}=\delta_{L}=0.5$ illustrated in Figure 5a. It would be interesting to know how far the results could be extended. 

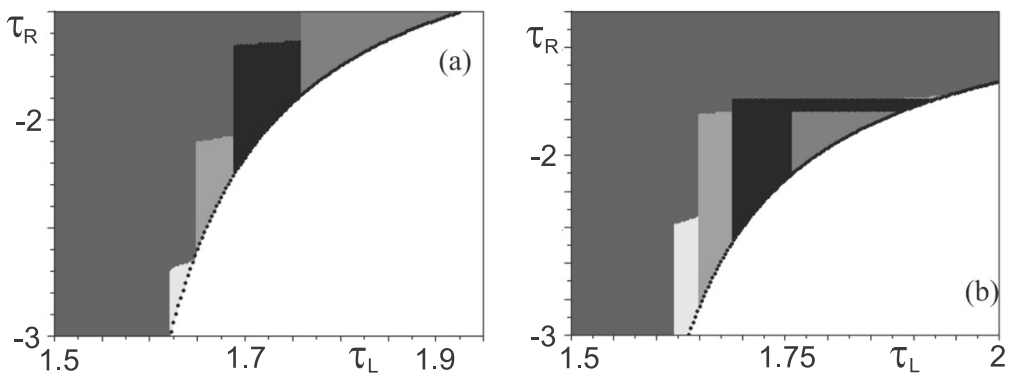

Fig. 5. $\left(\tau_{L}, \tau_{R}\right)$-parameter space for robust: there is a convex trapping region for parameters in the shaded areas, and the additional shading close to the boundary between shaded and non-shaded areas indicates those regions for which an invariant measure can be proved to exist with $N=3,4,5,6$ moving down the figures. In (a) $\delta_{R}=\delta_{L}=0.5$, so the issue of choosing $\varepsilon$ to be the larger of $\delta_{L}$ and $\delta_{R}$ creates no restrictions; in (b) $\delta_{L}=0.5$ and $\delta_{R}=0.2$ so we need to take $\varepsilon=\delta_{L}$.

\section{Robust chaos with two-dimensional attractors}

If at least one of the determinants $\delta_{k}$ has modulus greater than one a new possibility arises: the existence of strange attractors that have orbits dense in a two-dimensional region. If both determinants have modulus greater than one then the strong expansion means that to have attractors some folding is necessary and so the map cannot be a homeomorphism and $\delta_{L} \delta_{R}<0$.

Using the theoretical work of Buzzi [6] and Tsujii [23] it can be shown that there is an open set of parameters for the border collision normal form for which there is a two-dimensional strange attractor $[9,10]$. Moreover, there are open sets of the parameters $\left(\tau_{k}, \delta_{k}\right), k=L, R$, such that if $\mu<0$ the border collision normal form has a stable fixed point, whilst if $\mu>0$ it has a two-dimensional (robust chaos) chaotic attractor [11]. A numerical simulation of a two-dimensional attractor is shown in Figure 6 .

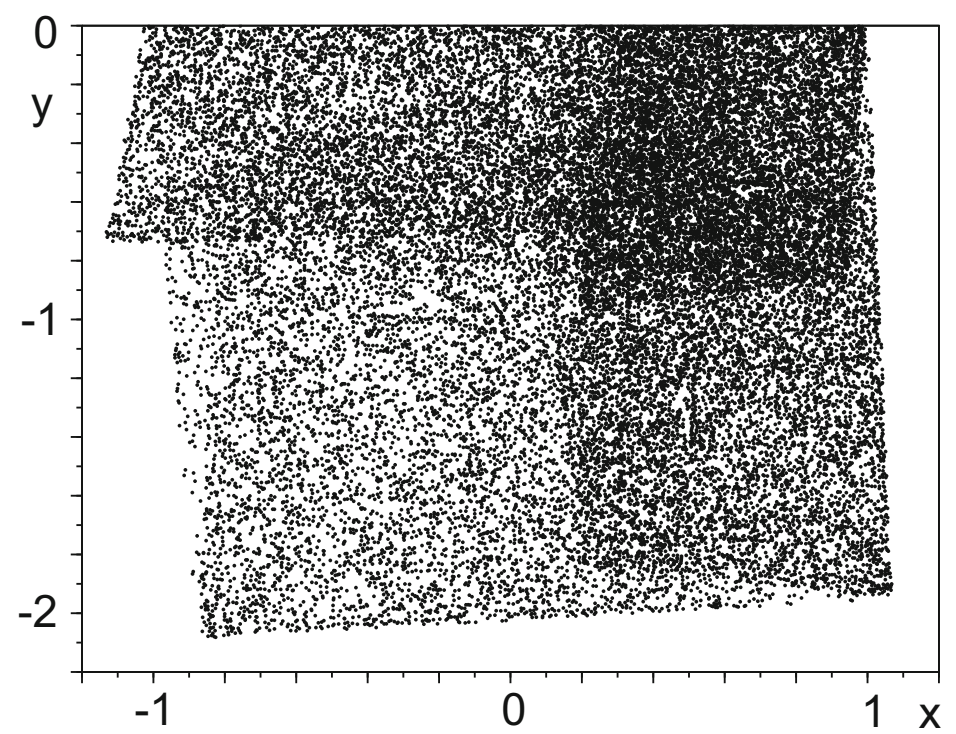

Fig. 6. 30000 iterations of an orbit on attractor computed numerically with $\delta_{L}=-0.85$, $\delta_{R}=1.95, \tau_{L}=0.06, \tau_{R}=0.07$. 
This poses an interesting question about the quasi-one-dimensional attractors for the area contracting cases described here: is it possible to have robust chaos in $\mu>0$ and a simple stable periodic orbit in $\mu<0$ ? If $\mu<0$ then a map $F \in B C N F_{R C}$ has no fixed points, so there can be no equivalent of the fixed point to two-dimensional attractor result. However, can there be stable periodic orbits in $\mu<0$ at the parameter values having robust chaos?

\section{Conclusion}

The concept of robust chaos provides an important way of looking at the attractors of piecewise smooth maps. Since it was introduced in [4] it has been applied many times, but there are still questions about the mathematical status of the examples cited (though no dispute about the phenomenon). In this paper I have tried to summarise what is known, adding an extra condition which is necessary, but may not be sufficient, for the phenomenon to occur in the border collision normal form via homoclinic intersections. I have also described other work that helps to understand the phenomenon, notably [16] and [24].

I have added a conjectured refinement to Young's Theorem as stated in [24] and verified the conditions of this Modified Young's Theorem on the computer, therefore (up to one's confidence in the algebraic evaluation of computers, the consistency of computer programmes, and Conjecture 7) confirmed the existence of robust chaos over a range of parameter values. I have also described how recent work on expanding maps implies the existence of robust chaos with two-dimensional attractors [9-11].

Inevitably when discussing open problems there is an element of personal prejudice here. I have tried to be clear about what I understand and what I do not understand, leaving some questions open for future work. I think effort on this problem is worthwhile. The phenomenon is real and interesting. A more thorough (or at least transparent) set of results would help our understanding of a broad range of problems in piecewise smooth dynamical systems.

This work was partially funded by EPSRC grant EP/E050441/1. I am grateful to the Simons Foundation for support during a stay at CRM, Barcelona, in 2016 where part of this work was done.

\section{References}

1. V.S. Afraimovich, N.I. Chernov, E.A. Sataev, Statistical properties of 2-D generalized hyperbolic attractors, Chaos 5, 238 (1995)

2. K.T. Alligood, T. Sauer, J.A. Yorke, Chaos: an Introduction to Dynamical Systems (Springer, Berlin 1996)

3. S. Banerjee, C. Grebogi, Border Collision Bifurcations in Two-Dimensional Piecewise Smooth Maps, Phys. Rev. E 59, 4052 (1999)

4. S. Banerjee, J.A. Yorke, C. Grebogi, Robust Chaos, Phys. Rev. Lett. 80, 3049 (1998)

5. M. di Bernardo, C. Budd, A.R. Champneys, P. Kowalczyk, Piecewise-smooth Dynamical Systems: Theory and Applications, Applied Mathematical Sciences, Vol. 163 (Springer, London, 2008)

6. J. Buzzi, Absolutely continuous invariant measures for generic multi-dimensional piecewise affine expanding maps, Int. J. Bifurc. Chaos 9, 1743 (1999)

7. P. Collet, Y. Levy, Ergodic Properties of the Lozi Mappings, Comm. Math. Phys. 93, $461(1984)$

8. L. Gardini, Some global bifurcations of two-dimensional endomorphisms by use of critical lines, Nonlinear Anal. 18, 361 (1992) 
9. P. Glendinning, Attractors with dimension $n$ for open sets of parameter space in the n-dimensional border collision normal form, Int. J. Bifurc. Chaos 24, 1450164 (2014)

10. P. Glendinning, Bifurcation from stable fixed point to $N$-dimensional attractor in the border collision normal form, Nonlinearity 28, 3457 (2015)

11. P. Glendinning, Bifurcation from stable fixed point to two-dimensional attractor in the border collision normal form, IMA J. Appl. Math. 81, 699 (2016)

12. M. Hénon, A two-dimensional mapping with a strange attractor, Comm. Math. Phys. 50, 69 (1976)

13. B.R. Hunt, J.A. Kennedy, T.-Y. Li, H.E. Nusse, SLYRB measures: natural invariant measures for chaotic systems, Physica D 170, 50 (2002)

14. R. Lozi, Un attracteur étrange du type attracteur de Hénon, J. Phys. (Paris) Coll. C5 39, 9 (1978)

15. Y. Maistrenko, I. Sushko, L. Gardini, About two mechanisms of reunion of chaotic attractors, Chaos Solitons Fractals 9, 1373 (1998)

16. M. Misiurewicz, Strange attractors for the Lozi mapping, Ann. NY Acad. Sci. 80, 348 (1980)

17. H.E. Nusse, J.A. Yorke, Border-collision bifurcation including 'period two to period three' for piecewise-smooth systems, Physica D 57, 39 (1992)

18. J. Palis, W. de Melo, Geometric Theory of Dynamical Systems (Springer, 1982)

19. D.J.W. Simpson, Bifurcations in Piecewise-Smooth Continuous Systems, World Scientific Series on Nonlinear Science Series A, Vol 70 (World Scientific, 2010)

20. D.J.W. Simpson, Unfolding homoclinic connections formed by corner intersections in piecewise-smooth maps, Chaos 26, 073195 (2016)

21. S. van Strien, One-parameter families of smooth interval maps: Density of hyperbolicity and robust chaos, Proc. Amer. Math. Soc. 138, 4443 (2010)

22. I. Sushko, L. Gardini, Horseshoe Construction in Noninvertible 2D Border Collision Normal Form, in preparation

23. M. Tsujii, Abolutely continuous invariant measures for expanding piecewise linear maps, Invent. Math. 143, 349 (2001)

24. L.S. Young, Bowen-Ruelle measures for certain piecewise hyperbolic maps, Trans. Amer. Math. Soc. 287, 41 (1985)

Open Access This is an Open Access article distributed under the terms of the Creative Commons Attribution License (http://creativecommons.org/licenses/by/4.0), which permits unrestricted use, distribution, and reproduction in any medium, provided the original work is properly cited. 